\title{
Mesolimbocortical dementia: clinico-pathological studies on two cases
}

\author{
M Anthony Verity, B Roitberg, J J Kepes
}

\begin{abstract}
The clinicopathological findings are presented of two cases of mesolimbocortical dementia. Both cases were characterised by late onset slowly progressive personality changes and progressive intellectual deterioration without clinical Parkinsonism. Neuropathological findings revealed non-specific neuronal degeneration, Holzer and GFAP positive gliosis primarily affecting the limbic system, caudate, thalamus and substantia nigra. The pathological findings coincide with the distribution of the non-striatal dopaminergic pathways and suggest an intrinsic involvement of these pathways to account for the clinical and pathological manifestations.
\end{abstract}

In 1986 Torack and Morris ${ }^{1}$ reported the clinical and pathological findings of a 57 year old woman, who gradually developed an illness characterised by progressive dementia, behavioural changes and Parkinsonism. The pathological process consisted of neuronal loss, non specific degenerative changes, gliosis affecting the limbic system and caudate nucleus, as well as the mesencephalic dopaminergic neurons and their cortical terminal fields. The term "mesolimbocortical dementia" was proposed for this putative new entity.

We report two further patients with dementia and behavioural changes in whom the neuropathological findings were similar to the case described by Torack and Morris. ${ }^{1}$ We suggest that our cases represent examples of mesolimbocortical dementia.

\section{Case 1}

A 78 year old right handed man had memory difficulty and depression. There was no family history of dementia and no history of alcohol use. At that time he was seen by a neurologist and a diagnosis of pseudodementia secondary to depression was made. Past medical history included surgery for right colon carcinoma two years earlier.

During the following three years he progressively deteriorated and became lost several times. On one of these occasions he was found lying in the street and was taken to hospital. On admission there was a suggestion of left sided weakness, but a subsequent evaluation by a neurologist did not confirm this. He was placed on dipyridamole.

Neurological and psychological evaluations were performed. The patient was alert, in no distress, but with a flat affect. $\mathrm{He}$ was able to partially take care of himself, but had forgotten basic skills such as turning off tap water or cutting food. He had limited short and long term memory, and followed one step directions only. The ability to sustain attention and concentration was poor and he was not participating in any kind of leisure activity. The patient was moderately impaired on a standardised test of orientation (Galveston Orientation and Amnesia Test, score 48) and was impaired on a test of ability to concentrate for a brief period of time (WAIS-R Digit Span Subtest and Wechsler Memory Scale Mental Control Subtest). His efficiency in learning new information and ability to retain it were both significantly impaired (Luria Nebraska Neuropsychological Battery Memory Subtest). Verbal expression was intact with the exception of an occasional difficulty in finding the appropriate word. General problem solving skills and ability to abstract were only minimally impaired for age (WAIS-R Arithmetic, Block Design, and Similarities Subtests). Further neurological examination was unhelpful. An EEG was performed at that time, which showed mild generalised disturbance of cerebral activity without focal or paroxysmal features. Audiological evaluation revealed moderate sensorineural hearing loss at higher frequencies.

One year later, at the age of 82 years, the patient returned for follow up. His memory had continued to deteriorate and there were episodes of urinary incontinence. Verbal output had declined, and he was mute much of the day. Behavioural changes were noted, which included withdrawal, careless appearance and increased impulsivity. His gait was on a broadened base with a suggestion of pedestal turning. The patient died four months later, of metastatic carcinoma with bowel obstruction.

\section{Neuropathological Studies}

Necropsy was limited to the brain. The brain weighed 1400 grams, and no gross abnormalities were found. The central finding on microscopic examination was limbic sclerosis. The hippocampus was atrophic. The granular neurons were well organised. Pyramidal neurons throughout $\mathrm{CA} 1$ and part of CA2 were lost (fig 1) with fibrillary and gemistocytic gliosis through CA1 into the subiculum and parahippocampal gyrus (fig 2). The molecular zone, situated between CAl and the granule layer revealed astrocytosis. 
Figure 1 Case 1. Coronal section of slightly atrophic hippocampus revealing rarefaction, neuronal loss and gliosis through CA2-CA1 (arrows).

(Haematoxylin-eosin $\times$ 12).

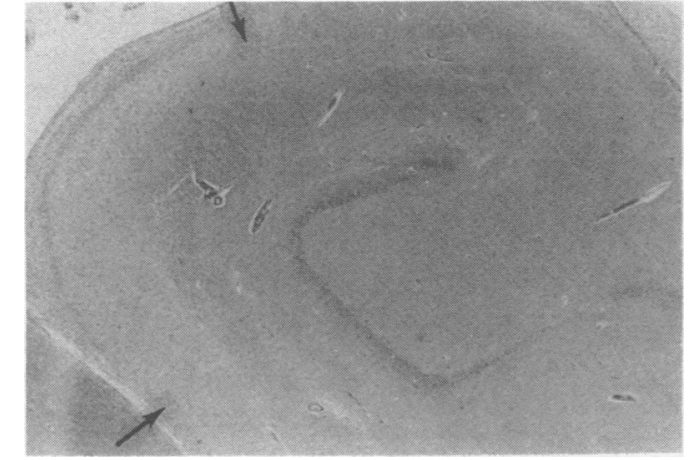

Figure 2 Case 1. Early, senile plaques throughout intermediate and deep neuronal laminae of inferior temporal cortex. No neurofibrillary tangles observed. (Bielschowsky silver stain, $\times 30$ ).

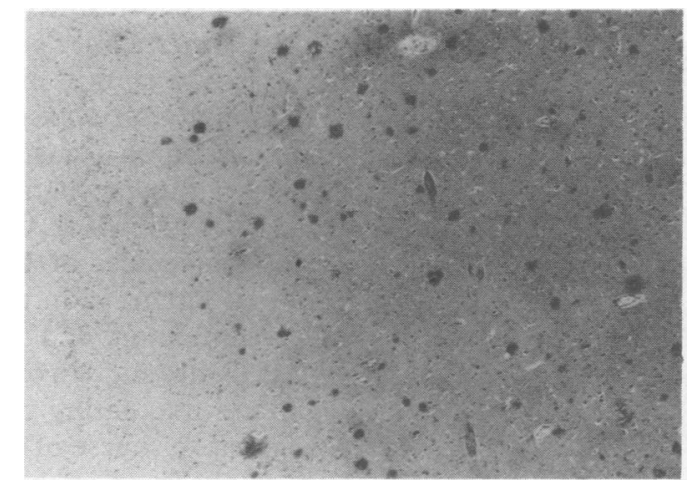

Gliosis was not evident in the adjacent inferior temporal cortex nor white matter. There were no argyrophilic plaques, neurofibrillary tangles, Pick bodies nor granulovacuolar change evident in the hippocampus. Primitive non-congophilic neuritic plaques were present in the temporal and parietal cortex (fig 2). Frontal, parietal, occipital and insular cortex revealed intact neuronal lamination, without achromasia or argyrophilic neurofibrillary tangle formations. The nucleus basalis of Meynert was intact. In the cingulate gyrus and corpus callosum there was minimal oedema of white matter and slight increase in astrocytes at the subcortical zone. The locus coeruleus was well pigmented without evidence of neuronal loss. There was moderate symmetrical gliosis of the inferior olives with focal neuronal drop-out. The head of the caudate nucleus revealed neuronal loss, glial nuclear hypertrophy, and GFAP reactive gliosis, especially in the inferior portion and as well as gliosis throughout the putamen.

The substantia nigra revealed multifocal neuronal degeneration and depigmentation with gliosis characterised by nuclear hypertrophy, and microglial proliferation (fig 3). Lewy

Figure 3 Case 1. Rare residual pigmented neurons surrounded by diffuse astrocytosis and microglial proliferation in substantia nigra. (Haematoxylineosin, $\times 120)$. bodies were not seen. The medial thalamus and subthalamic nuclei revealed patchy rarefaction, neuronal loss and severe gliosis with rare gemistocytic change.

\section{Case 2}

A 68 year old white woman developed progressive mental changes which included increasing forgetfulness, perseveration, urinary incontinence, and a glabellar reflex which did not extinguish. At age 70 years she was admitted to a nursing home. Due to behavioural problems, she had been given various antipsychotic drugs, most recently haloperidol. Medical history revealed chronic obstructive pulmonary disease of unknown duration and an oesophageal motility disorder. There was no history of alcohol use.

At age 71 years she was admitted to Kansas University Medical Center. She was confused, cyanotic and tachypnoeic. Her temperature was $39^{\circ} \mathrm{C}$. Bibasilar rales were present. Cranial nerves were intact, deep tendon reflexes were symmetric. There was no Babinski sign. ECG showed atrial fibrillation with a left bundle branch block and ventricular ectopic beats. Laboratory values on admission revealed WBC 18.4 (26 segs, 66 bands, 3 lymphocytes, 4 monocytes), platelets 478,000 , prothrombin time $13 \cdot 2$, blood urea nitrogen 44 , creatinine $1 \cdot 3$, glucose 158 .

The patient remained hypoxaemic and ultimately deteriorated despite treatment. She died on 11 March 1986, three years after the onset of her neurological illness.

\section{Necropsy and neuropathological findings}

Significant findings on general pathological examination included emphysema, severe bilateral bronchopneumonia, and ball thrombus in the right atrium.

The brain weighed $1080 \mathrm{~g}$. The dura and leptomeninges looked normal. There was minimal atrophy of the inferior temporal and frontal lobes and minimal atherosclerosis of the circle of Willis.

Microscopic examination revealed extensive loss of pyramidal neurons throughout the CA1 sector with sparing of Sommer's sector. Hypertrophic gemistocytic astrocytosis was present throughout CAl and extended into the subiculum (fig 4). The changes were more prominent in the anterior compared to the posterior hippocampus. Bielschowsky and Bodian silver stains did not show intraneuronal neurofibrillary change, senile plaques or granulovacuolar degeneration. The adjacent subiculum and parahippocampal cortex demonstrated marked atrophy, neuronal degeneration, laminar spongiosis and marked gliosis (fig 5). Similar gliosis was also present in the paraventricular (alveus and polymorphic neuronal) layer and amygdala. The inferior part of the caudate nucleus was atrophic with neuronal loss and gliosis which extended into the inferior putamen and white matter of the extreme capsule.

Free neuromelanin was noted in the substantia nigra, with minimal neuronal loss and mild 
Figure 4 Case 2. Neuronal disorganisation, superficial laminar spongiosis and extensive glial proliferation

throughout subiculum.

(Kluver-Barrera, × 30).

Figure 5 Case 2. Diffuse fibrillary and hypertrophic GF AP reactive gliosis in subiculum-parahippocampal cortex.

Immunoperoxidase for glial fibrillary acidic protein, $\times 130$ ).
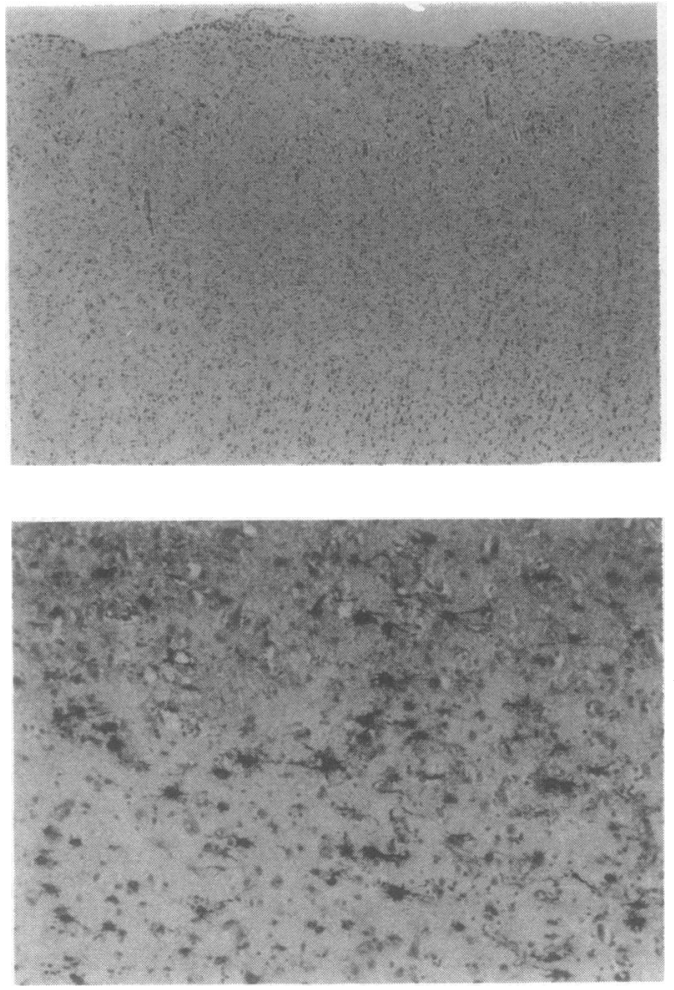

gliosis. The transverse section of the medulla through the inferior olives was unremarkable. The pons, locus coeruleus, cerebellum, and thalamus were normal.

\section{Discussion}

The neuropathological findings in our cases, summarised in the table and fig 2 were similar to the case reported by Torack and Morris, and are classified as mesolimbocortical dementia (MLCD). The clinical presentation of dementia was relatively non specific. Case 1 demonstrated relative sparing of problem solving and calculating functions, while memory, initiative and concentration were severely impaired, similar to that described by Torack and Morris, ${ }^{1}$ and considered to be a feature of subcortical dementia, in contrast to $\mathrm{Alz}$ heimer's disease. ${ }^{2}$

Our cases did not develop Parkinsonism, but both patients died early and the case reported by Torack and Morris ${ }^{1}$ did not develop Parkinsonism until late in the course of the disease. One of our patients (Case 1) exhibited a broad based, unsteady gait shortly before his death

Figure 6 A

Diagrammatic hemisection of cerebral hemisphere at the level of optic chiasma. B) Diagrammatic hemisection at level of thalamus. Blackened zones indicate areas of significant involvement.

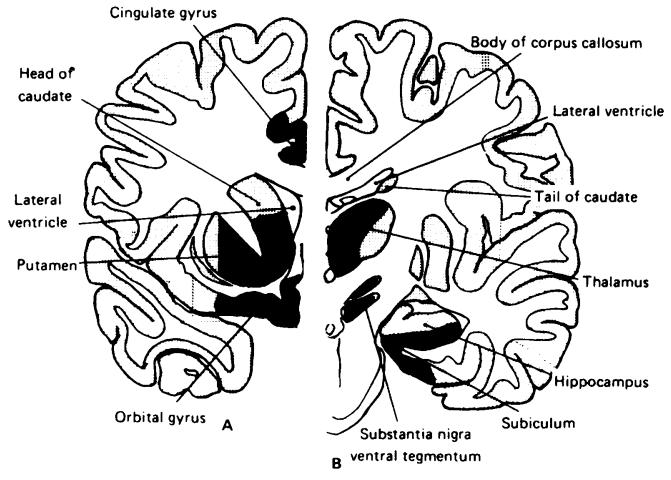

and revealed significant pathology in the substantia nigra.

Alzheimer's disease is the most common cause of dementia in the Western world, accounting for about $60 \%$ of all cases of dementia. ${ }^{34}$ The final diagnosis is only established at necropsy. The present cases were clinically diagnosed as dementia of Alzheimer type (DAT). Atrophy of the associative areas of the cortex, neurofibrillary tangles, cerebrovascular amyloid, granulovacuolar change, Hirano bodies, relative loss of large neurons especially of the nucleus basalis were absent in our cases and the neocortex was normal. It seems reasonable that Alzheimer's disease, ${ }^{3}$ was not responsible for the clinical picture.

Dementia on a vascular basis is the second most common cause of severe intellectual deterioration, accounting for $10-30 \%$ of all cases of dementia. ${ }^{34}$ Castaigne et al ${ }^{5}$ reported several instances of paramedial thalamic and midbrain infarcts, in which patients developed delayed memory and attention disturbances. It typically has sudden onset or step wise progression and fluctuating course, ${ }^{4}$ and not the slow progressive course of the degenerative dementia, as observed in the present cases. None of our cases had infarcts.

Selective involvement of the limbic system was described by $\mathrm{Kim} e t a l^{6}$ in a family in which four members developed dementia in middle or late life. Pathological examination revealed morphological alteration within the Sommer sector of the hippocampus in three cases. However, the findings were primarily that of cerebral atrophy, neuronal loss, gliosis and microcystic rarefaction, and relative preservation of the amygdala and parahippocampal cortex, all features notably absent in our cases. Herpes simplex encephalitis also has a predilection for limbic system structures. The term "limbic dementia" was coined to refer to the syndrome of amnesia and behavioural changes (denial of illness, distractability, change of affect) caused by extensive and selective destruction of the limbic system by $\mathrm{HSV}^{8}$ No suggestion of a necrotising inflammatory encephalitis was present here.

In 1960 Brierley et $a l^{9}$ described three cases of encephalitis, primarily involving the limbic system, one of which had remote carcinoma at necropsy. Corpus striatum, cerebellum and brainstem were variably involved. These cases probably represent examples of "limbic encephalitis with carcinoma", further defined by Corsellis et al..$^{10}$ Such patients presented with variable clinical findings including forgetfulness, changes in the level of consciousness, personality changes and sometimes focal neurological signs; ran a subacute course with maximal duration of sixteen months; had neuropathological findings of inflammation and degeneration of the limbic system, sometimes extending to the adjacent cortex, basal ganglia, or brainstem. Our Case 1 died with metastatic carcinoma. No brain metastases were found. The first signs of mental decline appeared two years after excision of a primary carcinoma of the colon and slowly progressed over the next five years. The diag- 
Table Distribution of neuronal loss and gliosis in mesolimbocortical dementia

\begin{tabular}{llll}
\hline & Case 1 & Case 2 & Torack and Morris \\
\hline Amygdala & - & ++ & ++ \\
Hippocampus & +++ & +++ & +++ \\
Subiculum & ++ & +++ & ++ \\
Parahippocampal cortex & ++ & +++ & +++ \\
Caudate (head) & ++ & +++ & ++ \\
Putamen & + & + & ++ \\
Thalamus & ++ & + & + \\
Substantia nigra & ++ & + & ++ \\
Orbital cortex & + & + & ++ \\
Cingulate & + & - & ++ \\
Prefrontal & 0 & 0 & + \\
Inferior olive & + & 0 & + \\
Neocortext, cerebellum, pons & 0 & 0 & ++ \\
\hline
\end{tabular}

Magnitude of neuron involvement and gliosis; - , tisssue not available for examination; 0 , normal + , mild; ++ , moderate; +++ , severe.

nosis of limbic encephalitis cannot be justified, on clinical or neuropathological grounds.

Progressive dementia has traditionally been associated with Alzheimer's disease. The concept that dementia may be part of the degenerative disorders involving subcortical structures is recent ${ }^{2}$ and dementia is now considered part of Parkinson's disease, Huntington's chorea, progressive supranuclear palsy, and multisystem degeneration. Numerous case reports of patients with dementia do not fit the commonly recognised categories. An early attempt to define a new entity was the identification by Neumann and Cohn ${ }^{11}$ of progressive subcortical gliosis. They reported four cases of progressive dementia with pronounced subcortical gliosis, without severe involvement of the cortex. The thalamus and brainstem were variably involved.

Verity and Wechsler ${ }^{12}$ described two further cases, reviewed the literature, and differentiated it from other forms of gliosis and dementia. Degeneration of the thalamus was found in the cases of progressive subcortical gliosis, in the case of MLC dementia ${ }^{1}$ and the present cases. Moossy et al ${ }^{13}$ reported two patients with dementia and thalamic gliosis combined with patchy astrocytosis of the neocortex. Adams et $a l^{14}$ described in four patients what they considered to be a variant of Parkinson's disease presenting clinically as Parkinsonian syndrome with variable mental and personality changes. The pathology consisted of striatonigral atrophy, loss of all neurons in the putamen and substantia nigra with variable involvement in the globus pallidus and caudate. The name "striato-nigral degeneration" was proposed for this entity but the involvement of the limbic system was minor.
We agree that mesolimbocortical (MLC) dementia is a rare degenerative disorder, characterised by neuronal loss and gliosis affecting structures of the limbic system, substantia nigra, caudate and inferior olivary nucleus. In common with other subcortical degenerative disorders, the pathological process is non specific with relative sparing of the neocortex. However, the unique topography of neuropathological findings merits a place within the spectrum of the subcortical dementias. The characteristic location of pathology to the subiculum, parahippocampal cortex, cingulate, orbito-frontal cortex and caudate nucleus, coincides with the non-striatal dopaminergic pathways. ${ }^{15}$ The absence of pathology in the nucleus basalis of Meynert and neocortex dissociates the cholinergic pathway and allows for conjecture that this pattern of dementia and its clinicopathological evaluation may reflect a primary disturbance in a dopaminergic system according to the spectrum of the dopaminergic dementias. ${ }^{16}$

1 Torack RM, Morris JC. Mesolimbocortical dementia. A clinicopathological case study of a putative disorder. Arch Neurol 1986;43:1074-8.

2 Huber SJ, Paulson GW. The concept of subcortical dementia. Am J Psychiatry 1985;142:1312-17.

3 Katzman R. Alzheimer's disease. $N$ Engl J Med 1986;314:964-73.

4 Mulley GP. Differential diagnosis of dementia. Br Med J 1986;292:1416-18.

5 Castaigne P, Lhermitte F, Buge A, et al. Paramedian thalamic and midbrain infarcts-clinical and neuropathological study. Ann Neurol 1981;10:127-48.

6 Kim RC, Collins GH, Parisi JE, Wright AW, Chu YB. Familial dementia of adult onset with pathological findings of a "non-specific" nature. Brain 1981;104:61-78.

7 Damasio AR, Van Hoesen GW. The limbic system and the localization of Herpes simplex encephalitis. $J$ Neurol Neurosurg Psychiatry 1985;48:297-301.

8 Gascon GG, Gilles F. Limbic dementia. J Neurol Neurosurg Psychiatry 1973;36:421-30.

9 Brierley JB, Corsellis JAN, Hierons R, Nevin S. Subacute encephalitis of later adult life. Mainly affecting the limbic encephalitis of later adult life.
areas. Brain 1960;83:357-68.

10 Corsellis JAN, Goldberg GJ, Norton AR. "Limbic encephalitis" and its association with carcinoma. Brain phalitis" and its

11 Neumann MA, Cohn R. Progressive subcortical gliosis-a rare form of presenile dementia. Brain 1967;90:405-17.

12 Verity MA, Wechsler AF. Progressive subcortical gliosis of Neumann: a clinicopathological study of two cases with review. Arch Gerontol Geriatr 1987;6:245-61.

13 Moossy J, Martinez J, Hanin I, et al. Thalamic and subcortical gliosis with dementia. Arch Neurol 1987;44:510-13.

14 Adams RD, Von Bogaert L, Van der Eecken H. Striatonigral degeneration. $J$ Neuropathol Exp Neurol 1964;23:584-608.

15 Lindvall O, Bjorklund A, Divac I. Organisation of mesencephalic dopamine neurons projecting to neocortex and phalic dopamine neurons projecting to neocortex and
septum. Adv Biochem Psychopharmacol 1977;16:39-46.

16 Whitehouse PJ. The concept of subcortical and cortical dementia: another look. Ann Neurol 1986;19:1-6. 